\title{
Transmitter and receiver solutions for VCSEL exploitation in access and metro networks
}

\author{
P. Parolari*a, A. Gatto ${ }^{a}$, M. Rapisarda ${ }^{a}$, F. Lipparini ${ }^{\mathrm{a}}$, C. Neumeyr ${ }^{\mathrm{b}}$, M. Svaluto Moreolo ${ }^{\mathrm{c}}$, P. Boffi ${ }^{\mathrm{a}}$ \\ aPolitecnico di Milano, Dip. Elettronica Informazione e Bioingegneria, Via Ponzio 34/5,20133 \\ Milano, Italy; ${ }^{b}$ VERTILAS GmbH, Daimlerstr 11d, D-85748 Garching, Germany; ${ }^{\circ}$ Centre \\ Tecnològic de Telecomunicacions de Catalunya (CTTC/CERCA), Av. C. F. Gauss 7, 08860 \\ Castelldefels (Barcelona), Spain.
}

\begin{abstract}
The high capacity demand, to support broadband services and everything-to-internet connectivity is pushing the limits of both access and metro networks, requiring the adoption of novel strategies for the optical transceiver modules. This represents an opportunity for the adoption in these network scenarios of novel photonic technologies based on single-mode vertical cavity surface emitting lasers (VCSELs) at long wavelengths. On one hand, the access network evolution requires a line rate increase beyond $10 \mathrm{~Gb} / \mathrm{s}$, targeting $50 \mathrm{~Gb} / \mathrm{s}$ propagation in passive optical networks (PONs) over a few tens of $\mathrm{kms}$ in standard single mode fiber (SSMF) with simple, cost effective and energy efficient transceivers. On the other hand, the future metropolitan area network (MAN) will need to handle multi- Tb/s traffic in a very dynamic scenario, over variable distances up to hundreds of kms while promoting sustainability, reducing the CapEx and OpEx costs and power consumption. Both needs can be fulfilled by adopting VCSEL direct modulation with multicarrier modulation formats such as discrete multitone (DMT), in combination with distinct transmitter architectures and receiver solutions to support different aggregate capacity requirements and transmission reaches. In any case DMT with bit/power loading enables flexible rate and adaptive distance for metro network applications and link adaptation and PON resource usage optimization for future access networks. In this work, we report our recent results on the adoption of VCSEL technology in both scenarios, with special focus on the receiver and transmitter adopted architectures.
\end{abstract}

Keywords: metropolitan area network, passive optical networks, discrete multitone modulation, vertical cavity surface emitting lasers, multi- $\mathrm{Tb} / \mathrm{s}$ capacty

\section{INTRODUCTION}

Nowadays, most of the total data traffic is concentrated in less of the $5 \%$ of the geographical area, this situation particularly impacts the operation of access networks, e.g. passive optical networks (PONs), and metropolitan area networks (MANs). The continuous increase of "hungry" applications and services such as 5G, Mobile Edge Computing, UHD TV, etc.. is generating a huge IP traffic which pushes the operational limits of PONs and MANs. To support this continuous growth of bandwidth demand coming from the core network both network segments need to adopt novel strategies.

After the not very successful experience of next generation (NG)-PON2 standard which tried to evolve the access topology introducing the wavelength dimension, obtaining a time and wavelength multiplexed (TWDM) -PON, it seems that access standardization is moving towards the line rate increase beyond the $10 \mathrm{~Gb} / \mathrm{s}$ of TDM-PONs. IEEE and ITU are looking for the candidate modulation formats and technologies for the next $50-\mathrm{Gbit} / \mathrm{s}$ PON $(50 \mathrm{G}-\mathrm{PON})^{1}$ : although it would be beneficial to rely on the pre-existence of mature high-volume technologies for data center intra-connect (DCI), PON links need to address much higher power budget and longer reaches with respect to point-to-point DCI optical links; thus, it is mandatory to adopt alternative solutions ${ }^{1}$. Moreover, network flexibility and link adaptation are becoming operators' requests because they offer several advantages such as a significant gain in the network capacity, the flexibility to adapt to high peak user rates and a more efficient usage of the network resources including energy consumption².

As MANs are concerned, beside the capacity increase, optical transport technologies should enable the development of agile solutions, different from the model of long-haul transmission, that can flexibly adapt to the path/traffic conditions,

*paola.parolari@polimi.it; phone 00390223998941 
associated with software defined networking (SDN), supporting a "pay-as-you-grow" evolution, to target cost reduction and possibly energy efficiency ${ }^{3}$.

In both segments, the answer to the above requirements can be given by the adoption of novel strategies for the optical transceiver modules, employing advanced photonic technologies based on single-mode (SM) vertical cavity surface emitting lasers (VCSELs) at long wavelengths (LW) and direct modulation (DM) with multicarrier modulation formats such as discrete multitone (DMT). Of course, the two network applications require distinct transmitter architectures and receiver solutions to support different aggregate capacity requirements and transmission reaches.

In the case of the access network, simplicity and low costs are required while targeting single wavelength $50 \mathrm{~Gb} / \mathrm{s}$ transceivers, thus direct detection (DD) is mandatory. Hence, as chromatic dispersion (CD) is a main impairment for 50GPON propagation, to target such rates over typical link distances of the order of $20 \mathrm{~km}$, the use of long wavelengths VCSELs in the O-band is necessary to take advantage of standard single mode fiber (SSMF) almost null CD.

In the case of MANs instead, the next generation transceivers should target multi-Tb/s capacities ${ }^{3}$, thus we propose to use massive photonic integration to develop a photonic integrated circuit (PIC) transmitter including multiple InP LW C-band high-bandwidth VCSEL sources and Silicon-photonic (SiPh) multiplexers ${ }^{4}$ to aggregate a multi- $\mathrm{Tb} / \mathrm{s}$ capacity ${ }^{5}$. The exploitation of DM with DMT modulation ${ }^{6}$ at the transmitter is combined with coherent detection (COHD) at the receiver side to allow propagation in hundreds of kilometers links.

In both cases DMT with bit/power loading enables a flexible rate, achieving a sliceable bandwidth and bitrate variable transceiver (S-BVT) able to adapt to metro network applications ${ }^{7}$ and link adaptation and PON resource usage optimization for future access networks.

In Section 2 we present the features characterizing the transceivers for the two scenarios, with particular regard to transmitter and receiver sides. In Section 3 we present results on $50 \mathrm{~Gb} / \mathrm{s} \mathrm{IM} / \mathrm{DD}$ downstream (DS) PON transmission and compare results with some simulations to achieve evaluations on the flexible PON aggregated capacity. Moreover, we experimentally assess the performance of DM-VCSELs in combination with COHD, exploiting DMT with single sideband (SSB) modulation to increase the spectral efficiency and target MAN distances and applications. Finally, Section 4 draws the conclusions.

\section{TRANSMITTERS AND RECEIVERS SOLUTIONS FOR PON AND MAN SCENARIOS}

As said previously, the common characteristics of our proposals for access and MAN scenarios is the exploitation of VCSELs directly modulated by multicarrier signals and in particular by $\mathrm{DMT}^{8,9}$.

VCSEL sources are characterized by a light emission normal to the semiconductor layer structure, that allows simple and cost-effective on-wafer test of VCSEL chips. The adopted LW InP VCSELs can show an emission wavelength up to 2 $\mu \mathrm{m}$; their design features a dielectric bottom mirror, a buried tunnel junction (BTJ) for the current confinement, a gold substrate acting as a heat sink, a multi quantum well (MQW) active region and an optimized waveguide design. SM operation is guaranteed by a transverse waveguide structure, with high sidemode suppression ratio and polarization stable output. A short cavity (SC) structure, displaying a very short resonator length and an optimized active region, reduces the effective photon lifetime while high relaxation-resonance frequencies and low parasitic effects guarantee high-speed modulations. SC devices present a current aperture of about $5 \mu \mathrm{m}$ and an effective cavity length of $2.5 \mu \mathrm{m}$ leading to modulation bandwidths up to $20 \mathrm{GHz}$ with relative intensity noise below $-140 \mathrm{~dB} / \mathrm{Hz}^{10}$. The use of VCSELs with DM and advanced modulation format can facilitate their exploitation for high speed transmission.

DMT with bit and power loading allows to adapt the transmitted signal to the link characteristics providing a great flexibility at the DSP level. In case of optical networks, this flexibility allows to optimize the transmitted capacity as a function of losses, transmission impairments (e.g. dispersion, nonlinearities etc.), and traffic patterns. At the transmitter digital-to-analog converters (DACs) are used to generate the DMT signal, which is fed to a laser linear driver; high-rate laser linear driver circuits are already commercially available for PAM4 applications ${ }^{11}$. At the receiver analog-to-digital converters (ADCs) are needed for further signal decoding. Due to the fast Fourier transform (FFT) and inverse FFT (IFFT) operations required to manage DMT signals, the needed relevant signal processing components are quite complex, but DSP-efficient FFT and simplified algorithms ${ }^{12}$ are being studied to simplify DMT use. We will analyze two DMT variants. The first one is dual side-band (DSB) DMT, in which the transmitted signal instantaneous power is modulated by a positive and real-valued DMT signal. The resulting optical spectrum is double-sided and requires a total bandwidth that is twice the bandwidth of the real DMT signal. The second DMT variant is single side-band (SSB) DMT, in which one of the two 
optical sidebands is removed by optical filtering achieving a better spectral efficiency. As subcarriers are loaded with multilevel QAM signals, $50 \mathrm{~Gb} / \mathrm{s}$ capacity can be easily obtained with a DMT modulating signal occupying a bandwidth of around $16 \mathrm{GHz}^{13}$. Although the same optical DMT signal is employed, different characteristics are necessary for both the transmitters and the receivers in the two scenarios.

\subsection{Access transmitter}

Transmitters for 50G PONs need to fit small form factors. Since the use of VCSEL simple direct DMT modulation avoids the need of an external modulator, the transmitter optical module, which is responsible for electro/ optical (E/O) conversion, is only constituted by the VCSEL itself and by the laser driver circuit. Considering that the main impairment in PON 50G transmission is CD, high-capacity downstream (DS) signals should be hosted in the O-band around $1342 \pm 2 \mathrm{~nm}^{14}$; yet, for a typical reach of $20-\mathrm{km} \mathrm{SSMF}$, the accumulated CD is around $74 \mathrm{ps} / \mathrm{nm}$. As said before, SC highbandwidth VCSEL technology is already reliable both in $\mathrm{O}$ and $\mathrm{C}$ bands; in fact, just the dielectric distributed Bragg reflectors differ between the two bands. Low-cost and energy-efficient transmitters for flexible 50G PON can be thus designed, considering that the VCSEL energy consumption per bit is about $0.7 \mathrm{pJ}$, while including the driver power consumption the total energy is about $5.3 \mathrm{pJ} / \mathrm{bit}$, that is pretty low with respect to the consumption of optical modules including standard DFB lasers and silicon photonics chips for external modulation ${ }^{15}$. In case of SSB DMT modulation obtained with optical filtering it is mandatory to stabilize the laser wavelength and, therefore, the power consumption of the transmitter nearly quadruplicates. On the other hand, application specific integrated circuit (ASIC) for DMT highbandwidth signals show an energy consumption around $50 \mathrm{pJ} / \mathrm{bit}^{16}$.

\subsection{MAN transmitter}

The target for MAN transmitters is to offer multi- $\mathrm{Tb} / \mathrm{s}$ capacity while allowing dynamic and flexible adaptation to traffic/channel conditions, achieving a multi-Tb/s sliceable bandwidth and bitrate variable transceiver (S-BVT) $)^{17}$. As said, flexibility is obtained through DMT modulation, while photonics technology can support the high-capacity target. In particular, multiple InP single-mode VCSELs, covering the whole C-band, can be massively integrated on a SOI chip obtaining a modular S-BVT transmitter, as depicted in Fig. 1. The single module is constituted by 40 VCSELs integrated with a $100-\mathrm{GHz}$ wavelength multiplexer providing up to $2 \mathrm{~Tb} / \mathrm{s}$ capacity; more details on the S-BVT architecture can be found in ${ }^{4,18}$. SSB modulation allows to interleave up to 4 identical SOI chip modules by temperature tuning to cover the C-band (about $4 \mathrm{THz}$ ) with $25-\mathrm{GHz}$ wavelength granularity ${ }^{19}$. Expecting a $50-\mathrm{Gb} / \mathrm{s}$ capacity per VCSEL per polarization, this super-module can therefore support up to $8 \mathrm{~Tb} / \mathrm{s}$ capacity. Polarization-division multiplexing (PDM) can be also exploited to double the total capacity obtaining up to $16 \mathrm{~Tb} / \mathrm{s}$ capacity per fiber with 4-b/s/Hz spectral efficiency ${ }^{20}$.
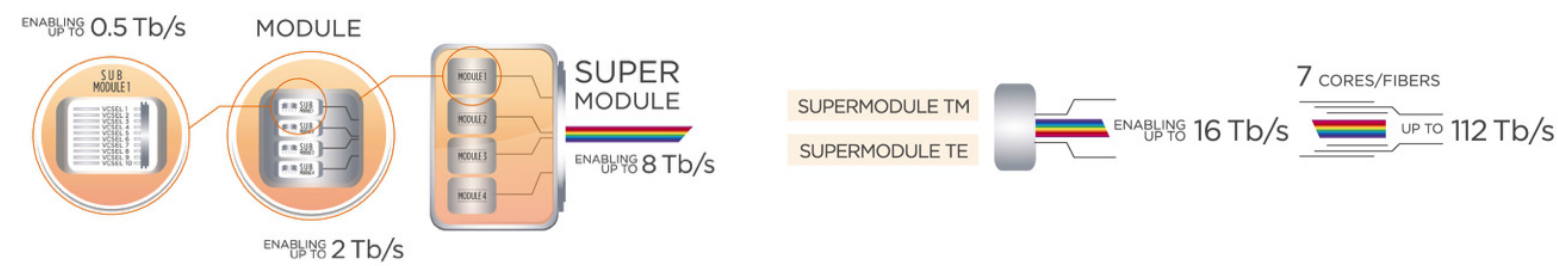

Fig. 1. Multi-Tb/s S-BVT transmitter based on VCSEL integration with modular approach.

\subsection{Access receiver}

In case of $50 \mathrm{G}$ PON, DD is the preferred receiver solution for its simplicity: the receiver block, in fact, is hosted in the ONU transceivers ${ }^{1}$. Indeed, thanks to DMT modulation the photodiode (PD) bandwidth can be around $25 \mathrm{GHz}$, allowing the exploitation of avalanche PD (APD) which are already commercially available ${ }^{1}$. The use of APD is fundamental to achieve the necessary power budget and support at least class N1, i.e. $29 \mathrm{~dB}$, ODN loss. Si-Ge APDs can achieve a gainbandwidth product of $300 \mathrm{GHz}$, which at $25 \mathrm{GHz}$ allows a gain of 12, with NEC around $20 \mathrm{pA} / \sqrt{\mathrm{Hz}}$ and responsivities close to $0.7 \mathrm{~A} / \mathrm{W}^{21}$.

\subsection{MAN receiver}

In order to target transmission distances typical of MANs, i.e. hundreds of kilometers, it is necessary to employ digital COHD which allows easy CD DSP compensation, avoiding also the VCSEL chirp interplay with CD. Moreover, COHD enables the use of PDM to increase the transmitted spectral efficiency. VCSEL DM leads to a transmitted signal which is just intensity modulated, thus a simplified coherent receiver can be used ${ }^{6}$ : after I and Q components recovery and CD 
compensation, the I and Q square moduli are performed and summed up in order to obtain the originally transmitted intensity signal. This approach has the drawback to cancel the COHD advantages in terms of bit error rate (BER) as a function of signal to noise ratio (SNR) with respect to DD, but avoids the use of optical carrier phase and frequency recovery, reducing the complexity of the receiver DSP and also relaxing the constraints on VCSEL and local oscillator (LO) linewidths ${ }^{22}$.

\section{ACCESS AND MAN SCENARIO EXPERIMENTAL RESULTS}

The above transmitters and receivers have been experimentally evaluated to demonstrate the effectiveness of the proposed architectures and photonic technologies. In both scenarios an 18-GHz Vertilas VCSEL is directly modulated by a DMT signal generated by a MICRAM 100-GS/s digital-to-analog converter (DAC10002) with 35-GHz electrical bandwidth and 6 bits vertical resolution. The DMT signal is calculated by Matlab ${ }^{\circledR}$ and is composed by 256 sub-carriers in $20-\mathrm{GHz}$ range, i.e. the sub-carrier spacing is $78.125 \mathrm{MHz}$. A cyclic prefix (CP) of about $2.1 \%$ of the symbol length is added. The VCSEL emitting wavelength is $1533.5 \mathrm{~nm}$, while its measured linewidth is about $5 \mathrm{MHz}$. The bias current is set to $9 \mathrm{~mA}$, with an optimized modulation amplitude of $10 \mathrm{~mA}$ and $8 \mathrm{~mA}$ for DSB DMT for SSB DMT modulations respectively. SSB modulation is performed by exploiting a programmable optical filter (Finisar WaveShaper 4000S) featuring a superGaussian transfer function in case of access scenario ${ }^{23}$, and the transfer function of 25-GHz standard WSSs in case of the MAN scenario ${ }^{24}$. A detuning of $8 \mathrm{GHz}$ with respect to the signal carrier, removing the low-frequency signal sideband, performs the single sideband filtering operation.

\subsection{G PON transmitters based on DM VCSELs}

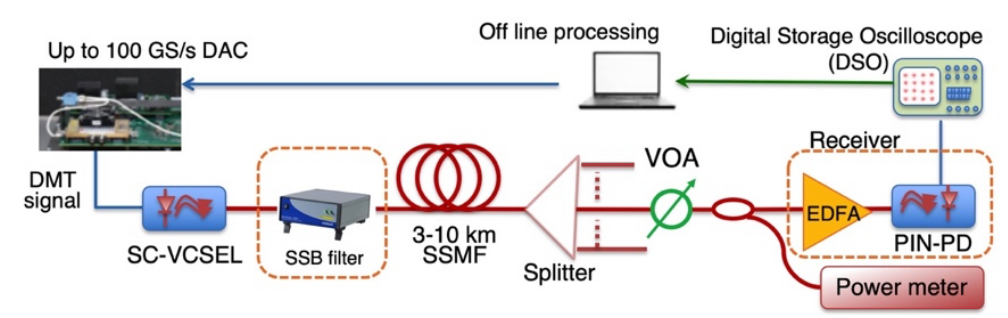

Fig.2 Experimental setup for 50G PON transceiver performance evaluation.

As depicted in Fig.2, the originated DMT signal is transmitted over different SSMF fiber spools, to evaluate CD tolerance of transmitters based on DM VCSELs accumulating a CD up to $160 \mathrm{ps} / \mathrm{nm}$. As the VCSEL under test emits at $1533.5 \mathrm{~nm}$, the target dispersion of $74 \mathrm{ps}$, equivalent to the cumulated CD of 20-km SSMF at $1342 \mathrm{~nm}$, is obtained with a spool of less than $6 \mathrm{~km} \mathrm{SSMF}{ }^{25}$. The effects of CD on the DM-DMT signal are evaluated up to 10-km SSMF propagation while a variable optical attenuator (VOA) takes into account further optical distribution network (ODN) losses. Both DSB and SSB DMT variants have been tested: in case of DSB DMT the programmable optical filter was removed after the VCSEL. At the receiver end, we used a preamplified receiver, composed by an Erbium-doped fiber amplifier (EDFA) followed by a $25-\mathrm{GHz}$ PIN photodiode, which can be anyhow substituted by a $25 \mathrm{G} \mathrm{APD}{ }^{1}$. The received signal is acquired by a Tektronix real-time oscilloscope (DPO 73304DX) with 8 bits vertical resolution, $100 \mathrm{GS} / \mathrm{s}$ and 33-GHz electrical bandwidth. Off-line processing provides digital symbol synchronization, CP removal, sub-carriers phase recovery, equalization and demodulation. Finally, the bit error rate (BER) count is obtained.

The evaluation of the performance is obtained by estimating at first the channel characteristics by transmitting a probe DMT signal with uniform QPSK loading, providing the signal-to-noise ratio (SNR) of each sub-carrier. Then, the measured SNRs are exploited for performing Chow's algorithm ${ }^{26}$, for a bit- and power-loading procedure by setting the target BER at $4.6 \cdot 10^{-3}$, as for advanced hard-decision forward error correction (FEC) code with $7 \%$ overhead $^{27}$.

Fig. 3 a) shows the measured transmitted capacities as a function of the received power for back to back (BTB), $50 \mathrm{ps} / \mathrm{nm}$ and $100 \mathrm{ps} / \mathrm{nm}$ cumulated CD. In BTB condition DSB modulation outperforms SSB modulation just for very high received optical powers (ROPs), while SSB modulation shows a better sensitivity to the ROP, permitting to achieve $50 \mathrm{~Gb} / \mathrm{s}$ even for $-24 \mathrm{dBm}$. For $50 \mathrm{ps} / \mathrm{nm}$ and $100 \mathrm{ps} / \mathrm{nm}$ cumulated dispersion conditions, SSB modulation has always better performance and achieves a sensitivity to target $50 \mathrm{~Gb} / \mathrm{s}$ transmission around $-23 \mathrm{dBm}$ for both propagation conditions, while DSB modulation requires $-18 \mathrm{dBm}$ and $-17 \mathrm{dBm}$, respectively. Considering that the VCSEL output power ranges around $7 \mathrm{dBm}$, the N1 class can be successfully supported for SSB modulation (circles, continuous line) up to $140 \mathrm{ps} / \mathrm{nm}$ as shown in Fig. $3 \mathrm{~b}$ ), allowing more than $35-\mathrm{km}$ propagation in O band. On the other hand, for DSB modulation (triangles, dashed line) the N1 class allows a capacity between 30 and $41 \mathrm{~Gb} / \mathrm{s}$. Finally, if we focus on the CD target of $74 \mathrm{ps} / \mathrm{nm}$, in Fig. $3 \mathrm{c}$ ) it can be seen that DSB modulation is able to target the transmission capacity of $50 \mathrm{~Gb} / \mathrm{s}$ up to $24 \mathrm{~dB}$ ODN losses, while 
SSB modulation bears 6-dB more losses, outperforming DSB modulation. The SSB modulation behavior is consistent with its higher resilience towards CD impairments.
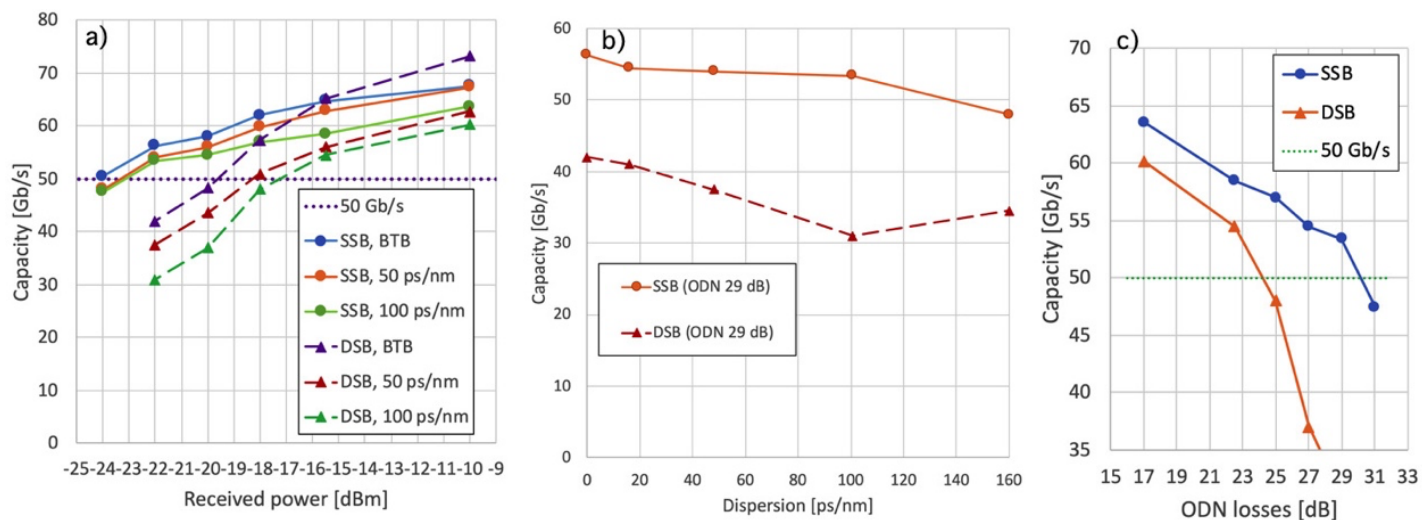

Fig. 3. a) Transmission capacities as a function of received power for SSB (circles) and DSB (triangles) DM-DMT VCSEL modulation for various accumulated CD. b) Transmission capacities for SSB (circles) and DSB (triangles) modulation as a function of cumulated $\mathrm{CD}$ for $23-\mathrm{dB}$ and $29-\mathrm{dB}$ ODN losses. c) Transmission capacities for SSB (circles) and DSB (triangles) modulation as a function of ODN losses for the target cumulated CD of $80-\mathrm{ps} / \mathrm{nm}$.

The demonstrated performance, when considering the statistical distribution of the ROP within one typical PON deployment ${ }^{2}, 28$, allows an increase in the throughput of a flexible PON employing the proposed DM-DMT VCSEL transmitter. For SSB DMT modulation the flexible VCSEL-based DS transmitter allows an aggregate rate of $\sim 58 \mathrm{~Gb} / \mathrm{s}, \sim$ $56 \mathrm{~Gb} / \mathrm{s}$ and $\sim 54 \mathrm{~Gb} / \mathrm{s}$ respectively for BTB, $50 \mathrm{ps} / \mathrm{nm}$ and $100 \mathrm{ps} / \mathrm{nm}$ cumulated CD. On the other hand, for DSB DMT modulation we obtain an aggregate rate of $\sim 46 \mathrm{~Gb} / \mathrm{s}, \sim 41 \mathrm{~Gb} / \mathrm{s}$ and $\sim 37 \mathrm{~Gb} / \mathrm{s}$ respectively for BTB, $50 \mathrm{ps} / \mathrm{nm}$ and 100 $\mathrm{ps} / \mathrm{nm}$ cumulated CD. In case of a $20-\mathrm{km}$ SSMF equivalent CD (around $70 \mathrm{ps} / \mathrm{nm}$ ), the flexibility allows a $10 \%$ and $50 \%$ increase for SSB and DSB DMT with respect to the fixed-rate 50G PON and 25G PON respectively ${ }^{29}$.

\subsection{VCSEL-based system exploiting DMT DM and COHD for multi-Tb/s metro link}
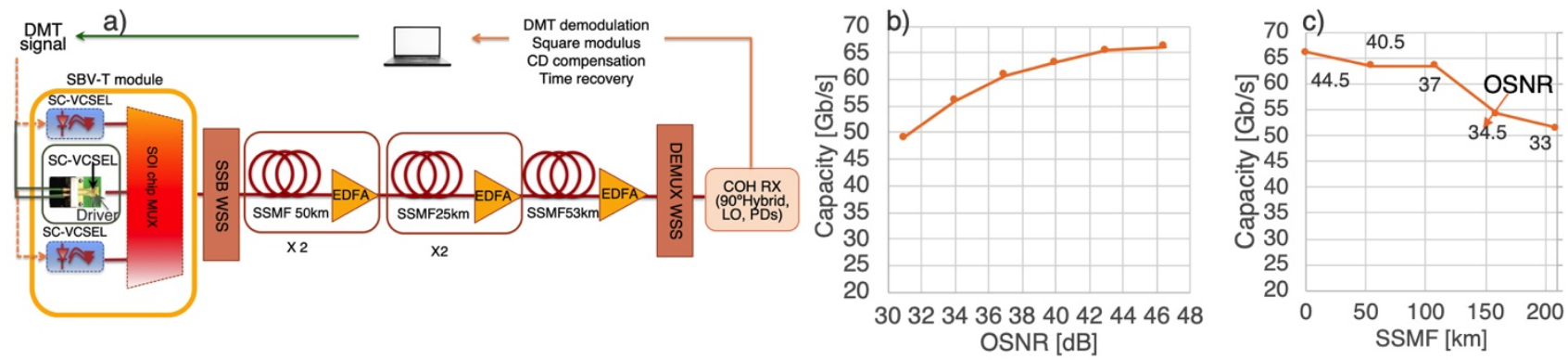

Fig.4 a) Experimental setup for MAN SBV-T performance evaluation. b) Total transported capacity vs OSNR in BTB condition. c) Total transported capacity vs SSMF propagation length for single-channel single-polarization transmission. The relative OSNR values measured at each SSMF reach are shown along the curve.

For the transmission performance evaluation in a MAN scenario we employ the experimental setup shown in Fig. 4a). Just the central single-polarization SSB optical signal is amplified by an Erbium-doped fiber amplifier (EDFA) and propagates on different SSMF spools with launch powers of $+1 \mathrm{dBm}$ and a total reach of about $200 \mathrm{~km}$ (implemented by amplified fiber spans with different lengths). The signal is detected by a Tektronix coherent receiver OM4245 with $45-\mathrm{GHz}$ bandwidth. The local oscillator (LO) is a tunable $100-\mathrm{kHz}$ laser with $+15.5 \mathrm{dBm}$ optical power. The inphase/ quadrature signals are acquired by a Tektronix real-time oscilloscope with 8-bits vertical resolution, 100-GS/s and 33-GHz electrical bandwidth respectively. The DSP functions described in paragraph 2.4 are performed off-line. The same performance as DD is obtained when the received optical power is enough to neglect the photoreceiver electrical noise. Furthermore, channel deskew, digital symbol synchronization, CP removal, sub-carriers phase recovery and demodulation are performed. The same procedure described in paragraph 3.1 is used for channel estimation, bit and power loading and error count. Fig. 4(b) shows the BTB performance in terms of total transported capacity as a function of the optical SNR (OSNR). As expected, the capacity increases with OSNR, ranging from about $50 \mathrm{~Gb} / \mathrm{s}$ for $31 \mathrm{~dB}$ OSNR to more than $66 \mathrm{~Gb} / \mathrm{s}$ for 
46 dB OSNR. Fig. 4(c) presents the capacities obtained after SSMF propagation for a single-channel single-polarization condition, while the measured OSNRs related to each SSMF reach for launch powers of $+1 \mathrm{dBm}$ are displayed below the curve. A capacity around $65 \mathrm{~Gb} / \mathrm{s}$ is maintained up to 100-km SSMF propagation thanks to high OSNR levels, while it decreases for $150 \mathrm{~km}$ and $200 \mathrm{~km}$. However, a total transported capacity per polarization higher than the target $50 \mathrm{~Gb} / \mathrm{s}$ can still be achieved even after $200-\mathrm{km}$ propagation. Finally, the cascade of WSS filters has been emulated by the programmable optical filter: the impact of the cascade of WSS filters, which can be detrimental due to the filter narrowing effect $^{30}$, is not significant. The capacity barely reduces increasing the number of crossed WSSs, allowing capacities higher than $50 \mathrm{~Gb} / \mathrm{s}$ even after 200-km SSMF propagation and 6 crossed WSS-like network nodes ${ }^{31}$.

\section{CONCLUSIONS}

As demonstrated by our recent results, the VCSEL technology adopted in specific transmitter and receiver architectures for PON and MAN scenarios can support the demanding requirements of future networks. In particular, DMT DM VCSELs achieving a per laser capacity beyond $50 \mathrm{~Gb} / \mathrm{s}$ are the building blocks for $50 \mathrm{G}$ PON cost effective and energy efficient transceivers supporting link adaptation and PON resource usage optimization. In combination with massive photonic integration they can otherwise support S-BVT handling multi-Tb/s traffic in a very dynamic scenario, with flexible rate and distance adaptation up to hundreds of kms.

\section{ACKNOWLEDGEMENT}

This work has been supported by the H2020 PASSION Project (GA 780326). The authors thank Tektronix, Micram and Opsys Technologies sponsorship.

\section{REFERENCES}

[1] V. E. Houtsma, D. T. van Veen, "Optical Strategies for Economical Next Generation 50 and 100G PON" in Optical Fiber Communication Conference OFC (2019), paper M2B.1.

[2] R. V. Der Linden, N. C. Tran, E. Tangdiongga and A. M. J. Koonen, "Increasing flexibility and capacity in real PON deployments by using 2/4/8-PAM formats," J. Opt. Commun. Netw., 9, A1-A8 (2017).

[3] M. S. Moreolo et al., " Spectrum/Space Switching and Multi-Terabit Transmission in Agile Optical Metro Networks," in OECC/PSC 2019 Proceedings, C000235.

[4] S. Bhat, et al. "Low Loss Devices fabricated on the Open Access 3mm SOI Waveguide Platform at VTT" in ECIO 2019, Ghent, Belgium, paper T.Po2.18 (2019).

[5] M. S. Moreolo et al, "Programmable VCSEL-based photonic system architecture for future agile Tb/s metro networks,” J. Opt. Commun. Netw. 13, pp. A187-A199, 2021.

[6] A. Gatto et al., "Discrete multitone modulation for short-reach mode division multiplexing transmission," Journal of Lightwave Technology, 37, 20, 5185-5192 (2019).

[7] M. Svaluto Moreolo et al., "Modular SDN-enabled S-BVT Adopting Widely Tunable MEMS VCSEL for Flexible/Elastic Optical Metro Networks," Proc. OFC, San Diego, CA (USA), pp. 1-3, 2018.

[8] D. Pilori, C. Fludger, and R. Gaudino "Comparing DMT Variants in Medium-Reach 100G Optically Amplified Systems". Lightwave Technol., 33, 3389-3399 (2016).

[9] A. Gatto, P. Parolari, C. Neumeyr, P. Boffi, "Beyond $25 \mathrm{~Gb} / \mathrm{s}$ directly-modulated widely tunable VCSEL for next generation access network", Proc. OFC 2018, San Diego, CA (USA), pp. 1-3, 2018.

[10] M. Mueller, W. Hofmann, T. Grundl, et al., "1550 nm high speed short-cavity VCSELs," IEEE J. Sel. Topics Quantum Electron., 17, 158-1166. (2011).

[11] https://www.idt.com/us/en/products/interface-connectivity/optical-interconnect-datacom/laserdrivers/hxt14100-56gbs-pam4-vcsel-driver accessed February 2020

[12] M. Chen, et.al, "Hardware-efficient implementation and experimental demonstration of Hermitian-symmetric IFFT for optical DMT transmitter," Opt. Express 27, 29817-29828 (2019).

[13] M. Rapisarda, et al. "Impact of Chirp in High-Capacity Optical Metro Networks Employing Directly-Modulated VCSELs," Photonics, 5, (4), pp. 51, 2018.

[14] M. Tao, J. Zheng, X. Dong et al., "Improved Dispersion Tolerance for 50G-PON Downstream Transmission via Receiver-Side Equalization," in Optical Fiber Communication Conference OFC (2019), paper M2B.3. 
[15] A. Boletti, P. Boffi, P. Martelli, M. Ferrario, and M. Martinelli, "Performance analysis of communication links based on VCSEL and silicon photonics technology for high-capacity data-intensive scenario," Optics Express, 23, 1806-1814 (2015).

[16] J. Wei, Q. Cheng, R. V. Penty, I. H. White and D. G. Cunningham, "400 Gigabit Ethernet using advanced modulation formats: Performance, complexity, and power dissipation," in IEEE Communications Magazine, vol. 53, no. 2, pp. 182-189, Feb. 2015.

[17] M. Svaluto Moreolo, et al. "SDN-enabled sliceable BVT based on multicarrier technology for multi-flow rate/distance and grid adaptation," J. Lightwave Technol., 34, 1516-1522, 2016.

[18]M. Svaluto Moreolo, et al., "VCSEL-based sliceable bandwidth/bitrate variable transceivers," Proc. SPIE Photonics West 2019, vol. 10946, paper 1094606 (2019).

[19] A. Gatto et al., "VCSEL-based communications for metro and access networks," in Proc. OECC/PSC 2018, paper 8751367, September 2018

[20]P. Boffi, et al., "Multi-Tb/s sustainable MAN scenario enabled by VCSEL-based innovative technological solutions", Proc. SPIE 11308, Metro and Data Center Optical Networks and Short-Reach Links III, 113080G (31 January 2020).

[21] M. Nada, T. Yoshimatsu, Y. Muramoto, H. Yokoyama and H. Matsuzaki, "Design and Performance of HighSpeed Avalanche Photodiodes for 100-Gb/s Systems and Beyond," J. Lightwave Technol., 33, 984-990 (2015).

[22]P. Parolari et al., "Effect of Filtering in Dense WDM Metro Networks Adopting VCSEL-Based Multi-Tb/s Transmitters," 2019 21st International Conference on Transparent Optical Networks (ICTON), Angers, France, pp. $1-4,2019$

[23] A. Malacarne, C. Neumeyr, W. Soenen et.al., "Optical transmitter based on 1.3- $\mu \mathrm{m}$ VCSEL and SiGe Driver Circuit for Short Reach Applications and beyond", J. Lightwave Technol., 36, 1527-1536 (2018).

[24] C. Pulikkaseril, et al., "Spectral modeling of channel band shapes in wavelength selective switches," Optics Express, 19, 8458-8470 (2011).

[25] T. Takahara, et al., "Discrete Multi-Tone for $100 \mathrm{~Gb} / \mathrm{s}$ optical access networks," in Optical Fiber Communication Conference OFC (2014), paper M2I.1, (2014).

[26]P. S. Chow et al., "A practical discrete multitone transceiver loading algorithm for data transmission over spectrally shaped channels," IEEE Trans. on Comm., 43, 773-775, 1995

[27] R. Rodes, et al., "High-Speed $1550 \mathrm{~nm}$ VCSEL Data Transmission Link Employing 25 GBd 4-PAM Modulation and Hard Decision Forward Error Correction," J. Lightwave Technol., 31, 689-695 (2013).

[28] V. E. Houtsma and D. T. Van Veen, "Investigation of Modulation Schemes for Flexible Line-Rate High-Speed TDM-PON," J. Lightwave Technol. 38, 3261-3267, 2020.

[29] P. Parolari, A. Gatto, C. Neumeyr and P. Boffi, "Flexible transmitters based on directly modulated VCSELs for next-generation 50G passive optical networks," in IEEE/OSA Journal of Optical Communications and Networking, 12, D78-D85, October 2020.

[30] J. M. Fàbrega et al., "On the filter narrowing issues in elastic optical networks," J Opt Commun Netw, 8, A23A33 (2016).

[31] A. Gatto et al., "Long-wavelength VCSEL-based system exploiting direct DMT modulation and coherent detection for multi-Tb/s metro link" in Proceedings 24th International Conference on Optical Network Design and Modeling, ONDM 2020, Castelldefels, Barcelona, Spain, May 18-21, 2020 